\title{
Coherent multiple light scattering in Faraday active materials
}

\author{
L. Schertel ${ }^{1,2}$, G.J. Aubry ${ }^{1, a}$, C. M. Aegerter ${ }^{2}$, and G. Maret ${ }^{1, b}$ \\ 1 Fachbereich Physik, Universität Konstanz, 78457 Konstanz, Germany \\ 2 Physik-Institut, Universität Zürich, Winterthurerstr. 190, 8057 Zürich, Switzerland
}

\section{Received 28 July 2016 / Received in final form 29 October 2016} Published online 25 May 2017

\begin{abstract}
Wave propagation in multiple scattering media shows various kinds of coherent phenomena such as coherent backscattering $[1,2]$ or Anderson localization [3], both of which are intimately connected to the concept of reciprocity. Manipulating reciprocity in such media is a powerful tool to study these phenomena in experiments [4]. Here we discuss the manipulation of reciprocity in reflection and transmission geometry for the case of light propagation in magneto-optical media. We show new experiments on coherent backscattering and speckle correlations in strongly scattering samples containing Faraday active materials $\left(\mathrm{CeF}_{3}\right)$ with transport mean free path in the $\mu \mathrm{m}$ range, at low temperatures $(T<10 \mathrm{~K})$ and high fields $(B=18 \mathrm{~T})$. Under such conditions we observe the effect of a Faraday rotation saturation in multiple scattering measurements.
\end{abstract}

\section{Introduction}

Coherence is a key concept in the field of wave transport in random media, such as sound wave propagation $[5,6]$ and matter wave scattering $[7,8]$. Neglecting coherence, one can describe the propagation of waves in random media as a diffusion process but fail to explain phenomena ranging from the celebrated Aharonov-Bohm effect for matter waves to ordinary speckle pattern of light waves (see for example [9]). Anderson localization [3] is the most studied coherent wave transport effect in the last 50 years. One contribution arises from constructive interference of reciprocal multiple scattering paths [10]. In the localized state the contribution of reciprocal paths to light transport become significant, which leads to a vanishing diffusion coefficient in an infinite medium [9]. Because Anderson localization is an interference effect, it should be affected if the relative phase between the reciprocal waves is perturbed. For light waves, the phase of the wave can be changed using the magneto-optical Faraday effect. This idea was indeed supported by the observation of the destruction of the coherent backscattering cone (CBC, which is often seen as the precursor of Anderson localization) in Faraday active multiple scattering samples [4,11]. While optical waves

\footnotetext{
a e-mail: Geoffroy.Aubry@uni-konstanz.de

b e-mail: Georg.Maret@uni-konstanz.de
} 
still bear to show signs of localization in 3D samples [12,13] Faraday rotation is still a good candidate for a sensitive probe of Anderson localization of light.

In this article, we present new experimental results on multiple light scattering in Faraday active materials by measuring the CBC and the speckles in transmission as in references $[4,11]$, but on samples scattering more strongly (although still in the weak localization regime). In particular, we compare the effect of the magnetic field on the CBC with its effect on the transmitted speckle in multiple scattering, slab shaped, samples. This shows how the magneto-optical Faraday effect in light transport depends on the geometry of the experiment. In the experimental range studied (high $B$ and low $T$ ) the Faraday active material (cerium fluoride, $\mathrm{CeF}_{3}$ ) shows a saturation effect. Therefore, in the last part of this article, we measure the transmitted speckle to perform a temperature and magnetic field study of the Faraday rotation of $\mathrm{CeF}_{3}$ in multiple scattering samples and compare it to measurements on bulk $\mathrm{CeF}_{3}$.

\section{Coherent backscattering and reciprocity}

Coherent backscattering was first explored with light waves $[1,2]$ although it is valid for all kinds of waves. In the case of light, photons traveling on time reversed scattering paths lead to an enhanced intensity in backscattering direction due to their constructive interference. Its origin is the reciprocity of light paths in multiple scattering samples.

In Faraday active multiple scattering samples, Lenke et al. [11] describe the field induced breaking of reciprocity of light transport by a simple model in which the CBC intensity $I(q, B)$ is a function of the backscattering vector $q(q=4 \pi \sin (\theta / 2) / \lambda$ with $\theta$ the backscattering angle (see Fig. $1, \theta=0$ and therefore $q=0$ for backscattering) and $\lambda$ the wavelength) and the magnetic field $B$ :

$$
I(q, B)=1+\frac{1-\exp \left[-2 \tilde{\gamma}_{R} q_{\mathrm{F}}(q, B) \ell^{*}\right]}{2 \tilde{\gamma}_{R} q_{\mathrm{F}}(q, B) \ell^{*}} .
$$

Here $q_{\mathrm{F}}(q, B)=\sqrt{q^{2}+2(V B)^{2}}, V$ is the sample's Verdet constant (a material constant which gives the strength of the Faraday rotation), $\ell^{*}$ is the transport mean free path (the characteristic scattering length after which the memory about the direction of the light flux is lost) and $\tilde{\gamma}_{R}=\frac{1+R}{1-R}(1+\gamma)$ is a factor related to the boundary condition, with $\gamma \approx 2 / 3$ the Milne parameter and $R$ the total reflectivity. The factor $\frac{1+R}{1-R}$ is the correction for internal reflections as calculated by Zhu et al. [14] which becomes relevant in our high refractive index samples.

Although more appropriate (but more complex) models are known (e.g. described in Ref. [15]) this simple description shows that the destruction of the cone tip intensity $I(q=0, B)$ with the field, and thus the breakdown of reciprocity, depends exponentially on the product of three parameters: the magnetic field $B$, the Verdet constant $V$ and the transport mean free path $\ell^{*}$. Since $V$ is fixed for a chosen material, temperature ${ }^{1}$ and wavelength, and $B$ is limited by the experimental setup, the dependency on $\ell^{*}$ sets the limits for the measurable range of the destruction of the CBC. For very strong scattering samples (small $\ell^{*}$ ), the cone tip will hardly be affected by Faraday rotation with the available magnetic fields.

The Faraday active material used is cerium fluoride (Sigma Aldrich $\mathrm{CeF}_{3}$ powder). $\mathrm{CeF}_{3}$ is a paramagnetic rare earth tri-fluoride that shows low absorption in the entire visible region where it has a refractive index of $n=1.6$ [18]. Crystalline bulk $\mathrm{CeF}_{3}$ has a high negative Verdet constant of $V=-1.1 \cdot 10^{3} \mathrm{rad} / \mathrm{T} \cdot \mathrm{m}$ at $\lambda=457 \mathrm{~nm}$ and

\footnotetext{
${ }^{1}$ For paramagnetic materials $V \propto 1 / T[16,17]$.
} 


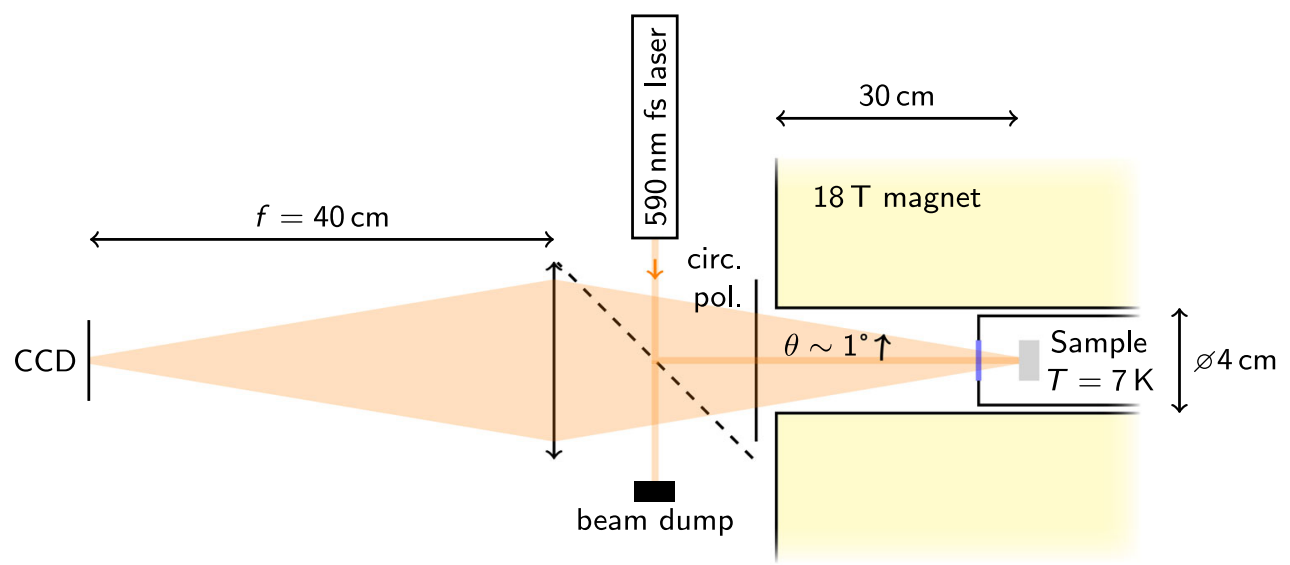

Fig. 1. CBC setup: a laser beam $(\lambda \approx 590 \mathrm{~nm})$ illuminates via a $50: 50$ beamsplitter a powder sample which is placed in a flow cryostat. The cryostat is located in a vertical bore $18 \mathrm{~T}$ magnet. A circular polarizer blocks single backscattered light. The reflected multiple scattered light is collected in $\mathrm{k}$-space on a CCD via a lens $(f=40 \mathrm{~cm})$ for scattering angles of up to $\theta \approx 1^{\circ}$.

$T=77 \mathrm{~K}[19] .^{2}$ To be able to change the scattering strength without changing the Faraday rotation strength, we embedded the $\mathrm{CeF}_{3}$ particles in glycerol $(n=$ $1.47[18])$ which is only weakly Faraday active $(V=3.87 \mathrm{rad} / \mathrm{T} \cdot \mathrm{m}$ at $\lambda=589 \mathrm{~nm}$ and $T=16{ }^{\circ} \mathrm{C}$ [18]) and added to this mixture a variable amount of titanium dioxide powder (anatase $\mathrm{TiO}_{2}$, Sigma-Aldrich, see [12]) which is diamagnetic and thus also weakly Faraday active even at low temperature $(V=100 \mathrm{rad} / \mathrm{T} \cdot \mathrm{m}$ at $\lambda=457 \mathrm{~nm}$ and $T=77 \mathrm{~K}[20])$. The mixtures were filled in slab shaped closed sample holders.

The coherent backscattering cone setup is similar to the one in reference [11]. This time, a superconducting magnet with a vertical room temperature bore (fields up to $B=18 \mathrm{~T}$, Oxford Instruments) was used. The slab shaped samples were placed with the sample holder inside an optical helium flow cryostat (Oxford instruments) to cool them down to $T \approx 7 \mathrm{~K}$. A beamsplitter underneath the magnet was used to send the light on the sample. A circular polarizer blocks single scattered light and allows for collection of multiple scattered photons in the helicity conserving channel. The reflected light was captured via a lens $(f=400 \mathrm{~mm})$ and led on a $1900 \times 1200$ pixel camera (The Imaging Source DMK 23UX174) placed in the focal plane of the lens. Figure 1 shows the CBC setup.

Since glycerol freezes out at low temperatures the scatterers are fixed in position leading to a static speckle pattern in backscattering. The backscattering cone is recovered by averaging over a large number of these different speckle patterns. This is done by turning the whole helium flow cryostat and tilting the sample inside the cryostat via piezo elements. We illuminated the sample with a fs-pulsed laser system at $590 \mathrm{~nm}$ (frequency doubled OPO pumped by a Ti:sapphire laser). The coherence length of this laser is so small that no static interference pattern coming from internal reflections in the cryostat windows and in the beamsplitter occurs, and even the sample's speckles are already a little bit averaged.

To show the destruction of the CBC in a magnetic field, we prepared a sample consisting of $26 \mathrm{vol} \% \mathrm{CeF}_{3}$ and $74 \mathrm{vol} \%$ glycerol. The transport mean free path measured outside the cryostat is $\ell^{*} \approx 58.9 \mu \mathrm{m}$, obtained by fitting the $\mathrm{CBC}$ with equation (1)

${ }^{2} V_{\mathrm{CeF}_{3}}(\lambda, T)=V_{\mathrm{CeF}_{3}}\left(\lambda^{\prime}, T^{\prime}\right) \frac{\lambda^{\prime 2}-\lambda_{0}}{\lambda^{2}-\lambda_{0}{ }^{2}} \frac{T^{\prime}}{T}$, where $\lambda_{0}=300 \mathrm{~nm}[19]$. 


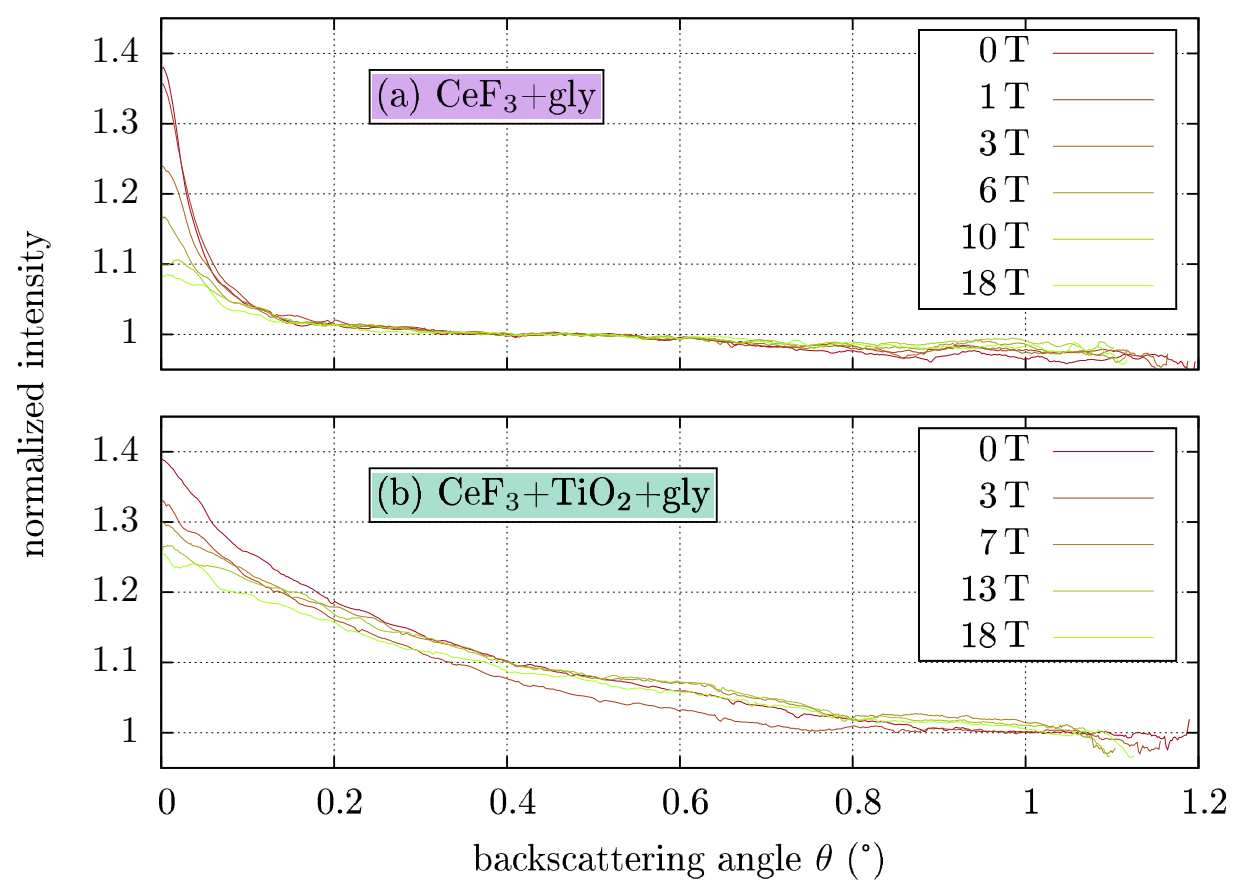

Fig. 2. CBC experiments for different magnetic fields between $0 \mathrm{~T}$ and $18 \mathrm{~T}$ for a sample consisting of (a) $26 \mathrm{vol} \% \mathrm{CeF}_{3}$ powder mixed with glycerol and (b) $26 \mathrm{vol} \% \mathrm{CeF}_{3}$ powder mixed with $2 \mathrm{vol} \% \mathrm{TiO}_{2}$ powder and glycerol. Experiments were carried out at $T=7 \mathrm{~K}$. Cones in (a) are normalized to a baseline between $0.4^{\circ}$ and $0.5^{\circ}$. Cones in (b) a normalized to a baseline between $1.0^{\circ}$ and $1.1^{\circ}$.

with $B=0 \mathrm{~T}$ and accounting for internal reflections from the sapphire window in front of the sample in the calculation of $R$. The field was then increased in several steps to $B=18 \mathrm{~T}$. Some of the cones measured are shown in Figure 2a, where a strong decrease of the enhancement of the CBC is observed with increasing field. For each cone, the enhancement factor was measured by dividing the intensity at $\theta=0^{\circ}$ by the mean intensity between $0.4^{\circ}$ and $0.5^{\circ}$ (where $I(q)$ is already flat, baseline). The measured enhancement at $B=0 \mathrm{~T}$ is about 1.4 , lower than 2 (the theoretical expected value) because of stray light and reflections from the windows of the cryostat. In Figure 3, these enhancement factors are plotted (violet curve) against the magnetic field for all measured cones. A parameter $\mathcal{N}$ is introduced to normalize the enhancement at $B=0$ to 2 . We used equation (1) at $q=0$ to fit the data. Due to the high fields and low temperatures, we are in a regime were the magnetization of $\mathrm{CeF}_{3}$ saturates with increasing field (see Figs. 6 and 7). For paramagnetic materials, $V$ is proportional to the magnetic susceptibility [16] which is the $B$-derivative of the magnetization. We assume therefore that it can be written for low temperatures [17] as

$$
V(B)=\frac{\kappa \alpha}{T}\left[1-\tanh ^{2}\left(\frac{\alpha}{T} B\right)\right]
$$

where $\kappa$ and $\alpha$ are material specific constants. $V_{0}=\kappa \alpha / T$ is the usual Verdet constant. The temperature $T$ is known from the measurement. Now we replace the phase $V B$ in equation (1) by

$$
\int_{0}^{B} V\left(B^{\prime}\right) \mathrm{d} B^{\prime}=\kappa \cdot \tanh \left(\frac{\alpha}{T} B\right)
$$




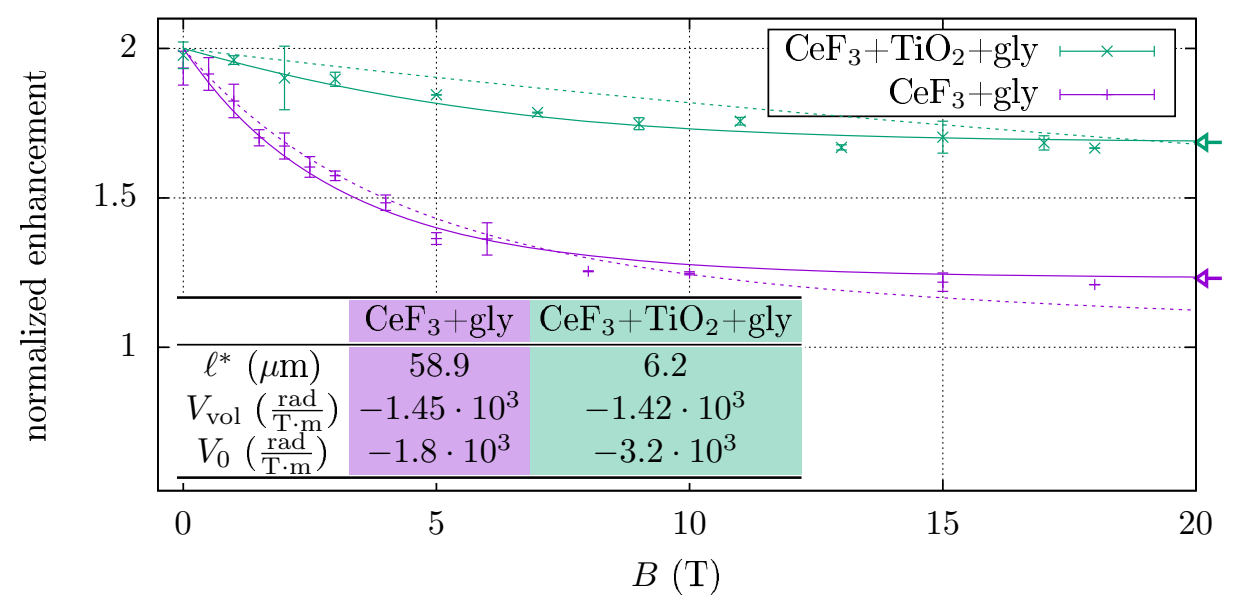

Fig. 3. Destruction of the CBC by a magnetic field: the enhancement $I(q=0)$ is shown for different field values for $\mathrm{CeF}_{3}$ mixed with glycerol (purple) and $\mathrm{CeF}_{3}$ mixed with $\mathrm{TiO}_{2}$ and glycerol (green). A fit (solid lines) with equation (1) and (3) is done to obtain the Verdet constant $V_{0}$. The data are then normalized by the second fit parameter $\mathcal{N}$ which takes into account that the experimental enhancement factor at $q=0$ is lower than 2 . The arrows on the right side indicate the lower bound of the enhancement factor due to $\mathrm{CeF}_{3}$ saturation. The decays of the CBC tip $I(q=0)$ expected from the volume fractions of the particles using equation (1) only are plotted as dashed lines for both samples. In both cases $V_{\mathrm{vol}}$ is calculated with $26 \mathrm{vol} \% \mathrm{CeF}_{3}$ at a temperature of $T=7 \mathrm{~K}$.

$\alpha$ is the parameter describing the saturation of the Faraday rotation. Because this saturation is only due to $\mathrm{CeF}_{3}$, we use the bulk value measured in Figure 6 . Finally $V_{0}$ (via $\kappa$ ) and $\mathcal{N}$ (the enhancement factor normalization parameter) are the two free parameters in equation (1) (modified with equation (3)) used to fit the data in Figure 3 (solid violet line). For this sample a value of $V_{0}=-1.8 \cdot 10^{3} \mathrm{rad} / \mathrm{T} \cdot \mathrm{m}$ is obtained, whereas the value calculated from the volume fraction of the particles is $V_{\mathrm{vol}}=(-1.45 \pm 0.07) \cdot 10^{3} \mathrm{rad} / \mathrm{T} \cdot \mathrm{m} .^{3}$

Mixing about the same amount of $\mathrm{CeF}_{3}$ and glycerol with $2 \mathrm{vol} \% \mathrm{TiO}_{2}$ (Aldrich Anatase) leads to lower $\ell^{*}\left(\ell^{*} \approx 6.2 \mu \mathrm{m}\right.$ compared to the $58.9 \mu \mathrm{m}$ of the sample without $\mathrm{TiO}_{2}$ ). The CBC of this sample are shown in Figure $2 \mathrm{~b}$ for different fields. These cones were normalized to a baseline between $1.0^{\circ}$ and $1.1^{\circ}$. The destruction of the cone is weaker than for the low-scattering sample, as shown in the green curve of Figure 3. Fitting the data gives a Verdet constant of $V_{0}=-3.2 \cdot 10^{3} \mathrm{rad} / \mathrm{T} \cdot \mathrm{m}$. For this sample, the value calculated from the volume fraction of the particles is $V_{\mathrm{vol}}=(-1.42 \pm 0.07) \cdot 10^{3} \mathrm{rad} / \mathrm{T} \cdot \mathrm{m}$.

The dashed lines on Figure 3 show the expected decorrelation with the respective $\ell^{*}$ and $V_{\text {vol }}$ without taking saturation into account (Eq. (1) only) for both samples. Erbacher et al. [4] observed a magnification $E_{V}=V_{0} / V_{\mathrm{vol}}>1$ of the Faraday rotation in multiple scattering samples, which they explain by internal reflections in the Faraday active particles. From this we expect the data to appear below the respective dashed curves from the volume fractions. This is the case for low magnetic field, whereas for higher magnetic fields the saturation of the Faraday rotation starts to play a role. Our fitted values of $V_{0}$ (Verdet constant at $B=0$ ) are higher than the volume fraction values $V_{\mathrm{vol}}$, in agreement with Erbacher et al. [4]. Note that their

${ }^{3} V_{\mathrm{vol}}=f_{\mathrm{CeF}_{3}} V_{\mathrm{CeF}_{3}}+f_{\mathrm{TiO}_{2}} V_{\mathrm{TiO}_{2}}$, with $f_{x}$ the volume fraction and $V_{x}$ the bulk Verdet constant. The error is calculated from the error of powder masses. 
experiments took place at higher temperatures and lower fields. This motivated us to fit our data with saturation (Eq. (3)).

Without saturation, it is theoretically possible to destroy the CBC completely (the normalized enhancement factor decreases toward 1 for large magnetic fields). However, saturation of the Faraday rotation defines a lower bound to the enhancement factor. Even with arbitrary large magnetic field, the normalized enhancement factor is limited to a lower bound of 1.2 (resp. 1.7) for the weakly (resp. strongly) scattering sample (see arrows in Fig. 3).

We confirm here earlier findings $[4,11]$ that the $\mathrm{CBC}$ can be destroyed in high magnetic fields in multiple scattering Faraday active samples. We extend the range of experimental conditions to higher Verdet constants and lower $\ell^{*}$. Moreover, we show here the effect of a saturation on the CBC destruction.

\section{Speckle correlations in transmission}

The strength of the Faraday rotation in multiple scattering samples can also be quantified by measuring the decorrelation of the transmission speckle with an applied magnetic field. The propagation along the magnetic field leads to an effective change of the photons phase, ending in a change of the speckle pattern for different applied fields. The theory of Faraday rotation in multiple scattering media is described in detail in reference [4]. A decay of the speckle intensity correlation function $C(B)=g_{2}(B)-1$, with $g_{2}(B)=\frac{\langle I(0) I(B)\rangle}{\langle I(0)\rangle^{2}}$ can be used to quantitatively measure the strength of the Faraday rotation and thus the Verdet constant $V$, via the field auto-correlation function [21]

$$
g_{1}(B)=\sqrt{C(B)}=\frac{\sinh \left(\tilde{\gamma} \ell^{*} \sqrt{\frac{(V B)^{2}}{2}+\left(\frac{1}{L_{\mathrm{a}}}\right)^{2}}\right) \sinh \left(\frac{L}{L_{\mathrm{a}}}\right)}{\sinh \left(L \sqrt{\frac{(V B)^{2}}{2}+\left(\frac{1}{L_{\mathrm{a}}}\right)^{2}}\right) \sinh \left(\tilde{\gamma} \frac{\ell^{*}}{L_{\mathrm{a}}}\right)} .
$$

$\tilde{\gamma}=1+\gamma=5 / 3$ is the boundary condition factor and $L_{\mathrm{a}}$ the macroscopic absorption length.

In contrast to the decay of the CBC with the magnetic field, this decay only weakly depends on the scattering length $\ell^{*}$, but is dominated by the sample thickness $L$ : it is governed by $\exp (-V B L)$ for low absorbing samples (large $L_{\mathrm{a}}$ ). This difference between this technique and the $\mathrm{CBC}$ is a consequence of the different path length distributions in reflection and transmission geometry. This effect can now be used to quantify the Faraday rotation also in strongly scattering samples with relatively low $\ell^{*}$ values, which are hard to measure in CBC experiments because of the Faraday rotation saturation discussed above.

Erbacher et al. [4] showed the validity of their model in experiments on milled paramagnetic Faraday glass (FR5 Hoya), with relatively large $\ell^{*} \approx 117 \mu \mathrm{m}$ values. The powder of Faraday active material was suspended in glycerol or paraffin to overcome speckle stability problems. In fact, one needs to stabilize the setup to ensure that the decorrelation only comes from the Faraday rotation and not from any vibration or particle displacement. They used a $23.5 \mathrm{~T}$ resistive magnet and cooled their samples to $T=30 \mathrm{~K}$ and $T=90 \mathrm{~K}$ in a flow cryostat.

In new experiments we use $\mathrm{CeF}_{3}$ and mix it with $\mathrm{TiO}_{2}$ (DuPont R700, see Ref. [12] for specs) to reach a stronger scattering regime. To overcome any change of the speckle by displacement of the particles in the applied field we embed the particles in a matrix of glycerol. With this we are able to repeat for the first time 


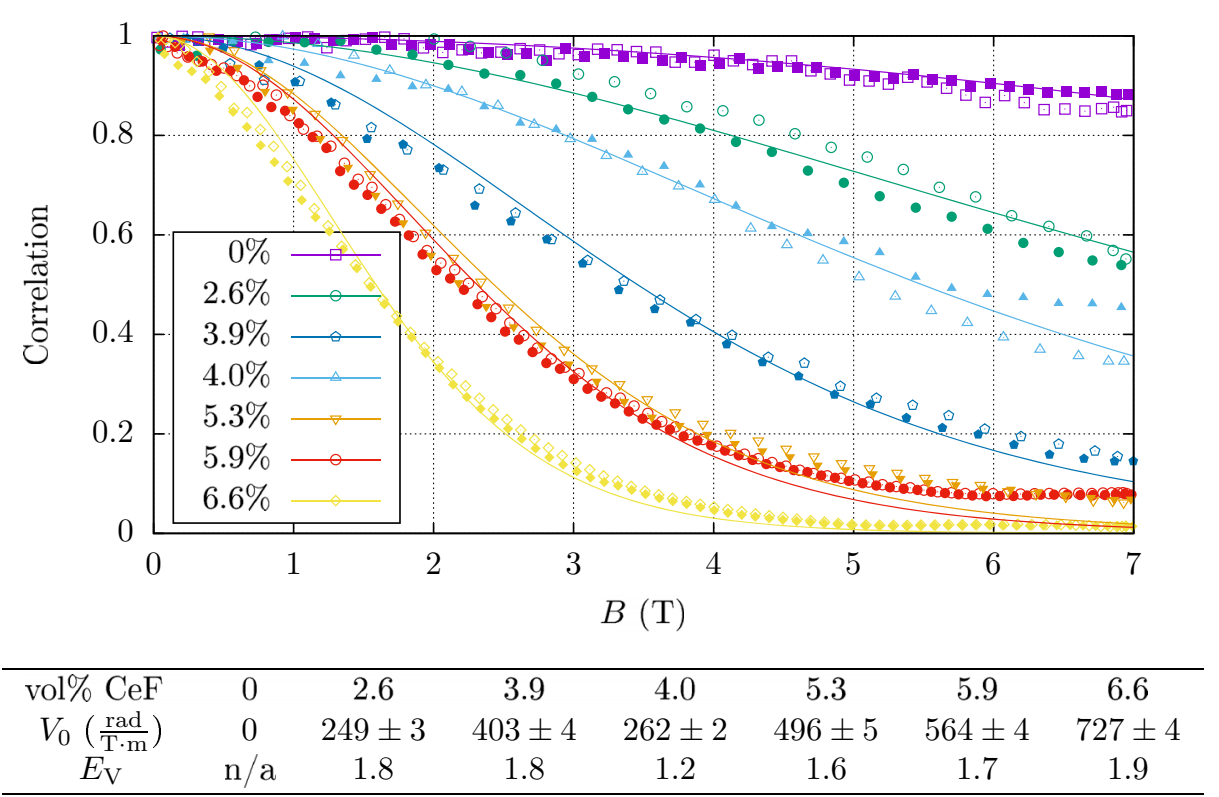

Fig. 4. Decorrelation of the transmission speckle pattern as a function of the magnetic field for different Faraday active powder mixtures solved in glycerol. The amount of $\mathrm{CeF}_{3}$ was raised from $0 \mathrm{vol} \%$ to $6.6 \mathrm{vol} \%$. Open symbols: increasing field, correlation with the first image; filled symbols: decreasing field, correlation with the last image. The corresponding fits (Eq. (4) with Eq. (3)) with $V_{0}$ as the only free parameter are plotted as solid lines. In the fits we accounted for the Faraday rotation of the windows, the instability of each measurement (see footnote 4) and the saturation of the Faraday rotation (see Fig. 6). For each sample the increasing and decreasing field curves are fitted together. All measurements were carried out at $T=9 \mathrm{~K}$.

Erbacher et al. measurements, and extend the experimental conditions down to $\ell^{*} \approx 2 \mu \mathrm{m}$ and up to $B=7 \mathrm{~T}$.

A speckle interferometer setup was used to measure the transmission speckle of a multiple scattering sample. A CW-laser at $\lambda=532 \mathrm{~nm}$ illuminated the powder sample $(L=2.2 \mathrm{~mm})$, which was placed in an optical flow cryostat to cool it to low temperatures $(T<10 \mathrm{~K})$. The cryostat was fixed in a superconducting magnet with a room temperature horizontal bore which is able to generate magnetic fields up to $B=7 \mathrm{~T}$. The transmitted light was measured with a CCD-camera (Apogee Alta $\mathrm{U} 4000,2048 \times 2048$ pixel cooled at $-30^{\circ} \mathrm{C}$ ) after passing a linear polarizer which selects one plane of polarization. This is necessary since the model is calculated for one polarization plane only. The stability of the setup was intensively tested without and with magnetic field (see Ref. [22]). ${ }^{4}$

To show that $\mathrm{TiO}_{2}$ and glycerol have a negligible Faraday rotation we measured $\mathrm{TiO}_{2}$ in glycerol only (see violet curve in Fig. 4). The observed decay can be completely accounted for by the Faraday rotation of the cryostat windows, which was measured separately to be $V_{\mathrm{w}}=(3.00 \pm 0.04)^{\circ} / \mathrm{T}$. The decay of $C(B)=\cos ^{2}\left(V_{\mathrm{w}} B\right)$ expected from the windows is plotted as a solid violet line in Figure 4 (no fit).

${ }^{4}$ Before each run the stability of the speckle was measured during a time as long as the field sweep duration and fitted by a quadratic polynomial. The correlation functions during the field sweeps were then divided by the polynomial to take into account the decorrelation due to the setup instability. 
A series of measurements of the Verdet constant of Faraday active powder mixtures consisting of $\mathrm{CeF}_{3}$ and $\mathrm{TiO}_{2}$ solved in glycerol was carried out. The amount of $\mathrm{CeF}_{3}$ was raised from $0 \mathrm{vol} \%$ to $6.6 \mathrm{vol} \%$ in the sample. The correlations of the transmission speckle as function of the magnetic field are plotted in Figure 4. The decay gets larger when increasing the amount of $\mathrm{CeF}_{3}$. While the Faraday rotation is dominated by the added amount of $\mathrm{CeF}_{3}$, the scattering behavior (and thus $\ell^{*}$ ) scales with the added amount of $\mathrm{TiO}_{2}$ (which was varied between $0 \mathrm{vol} \%$ and $6 \mathrm{vol} \%$ ), as observed in time of flight experiments [22]. All fits of the intensity auto-correlation function are shown as solid lines in Figure 4. Increasing and decreasing field data are fitted together. The fits were done using equation (4) again replacing $V B$ by equation (3) accounting for a saturation of the Faraday rotation using the bulk value for $\alpha$ (Fig. 6). Thus the $B=0 \mathrm{~T}$ Verdet constant $V_{0}$ is the only free parameter.

As an example, the sample consisting of $1.9 \mathrm{vol} \%$ (16.5 wt\%) $\mathrm{TiO}_{2}$ and $6.6 \mathrm{vol} \%$ (83.5 wt\%) $\mathrm{CeF}_{3}$ solved in $91.5 \mathrm{vol} \%$ glycerol is shown as yellow diamonds in Figure 4. Its scattering strength $\ell^{*} \approx 6 \mu \mathrm{m}$ was characterized in a CBC setup separately. The absorption length $L_{\mathrm{a}} \approx 750 \mu \mathrm{m}$ was extracted from time of flight experiments (see Ref. [22]). The same characterization was done for each sample.

The measured Verdet constants $V_{0}$ for the different samples are higher than expected from the volume fractions of $\mathrm{CeF}_{3}\left(V_{\mathrm{vol}}\right)$. In the $\mathrm{CBC}$ experiments $V_{0}$ was also higher than $V_{\text {vol }}$. Internal reflections in the Faraday active particles, which are not taken into account in the model of Erbacher might cause an underestimation of the Verdet constant, also observed by Erbacher et al. [4]. In their model, the correlation length of the Faraday rotation $\ell_{\mathrm{FR}}^{*}$ is assumed to be equal to the correlation length of the photon transport $\ell^{*}$. Lenke et al. [23] showed a scatterer size dependency, where the ratio $E_{V}=\ell_{\mathrm{FR}}^{*} / \ell^{*}$ decreases from 2 for Rayleigh scatterers to 1 with increasing scatterer size. This gives a physical explanation of the underestimation of the Faraday rotation in such multiple scattering samples. Erbacher et al. [4] obtained values around $E_{V}=2.2-2.6$ in their experiments using milled Hoya FR5 $(n=1.7)$ embedded in glycerol. In our case, we use $\mathrm{CeF}_{3}$ in glycerol, and thus the refractive index mismatch responsible for these internal reflections in the Faraday active particles is smaller. This might explain our smaller $E_{V}=1.2-1.9$ values.

Nevertheless, with this series of experiments we showed that the Erbacher model can be used to quantify the Faraday effect in multiple scattering samples measuring the transmission speckle correlation even for strongly scattering samples if the speckle stability is well controlled.

\section{Characterization of the Faraday rotation of $\mathrm{CeF}_{3}$}

In this last section, we use the speckle correlation technique described in Section 3 to characterize the Faraday rotation of $\mathrm{CeF}_{3}$ in multiple scattering samples as a function of temperature and field.

\subsection{Temperature dependency of the Faraday rotation of $\mathrm{CeF}_{3}$}

A sample consisting of $3.8 \mathrm{vol} \% \mathrm{TiO}_{2}$ and $5.3 \mathrm{vol} \% \mathrm{CeF}_{3}$ solved in $90.9 \mathrm{vol} \%$ glycerol was measured at $6 \mathrm{~K}, 9 \mathrm{~K}, 15 \mathrm{~K}, 30 \mathrm{~K}$ and $72 \mathrm{~K}$ to measure the temperature dependency of the Verdet constant of $\mathrm{CeF}_{3}$. This is shown in Figure 5. A scattering mean free path of $\ell^{*}=2.3 \mu \mathrm{m}$ was measured in a CBC experiment. The correlation curves show an increasing Faraday rotation with lower temperatures as expected from a paramagnetic material. At $T=72 \mathrm{~K}$ the measurement was less stable due to the high temperature. At this temperature, the cryostat was cooled with liquid nitrogen instead of helium. From the fitted Verdet constant $V_{0}$ (Fig. 5b) we extracted the bulk 


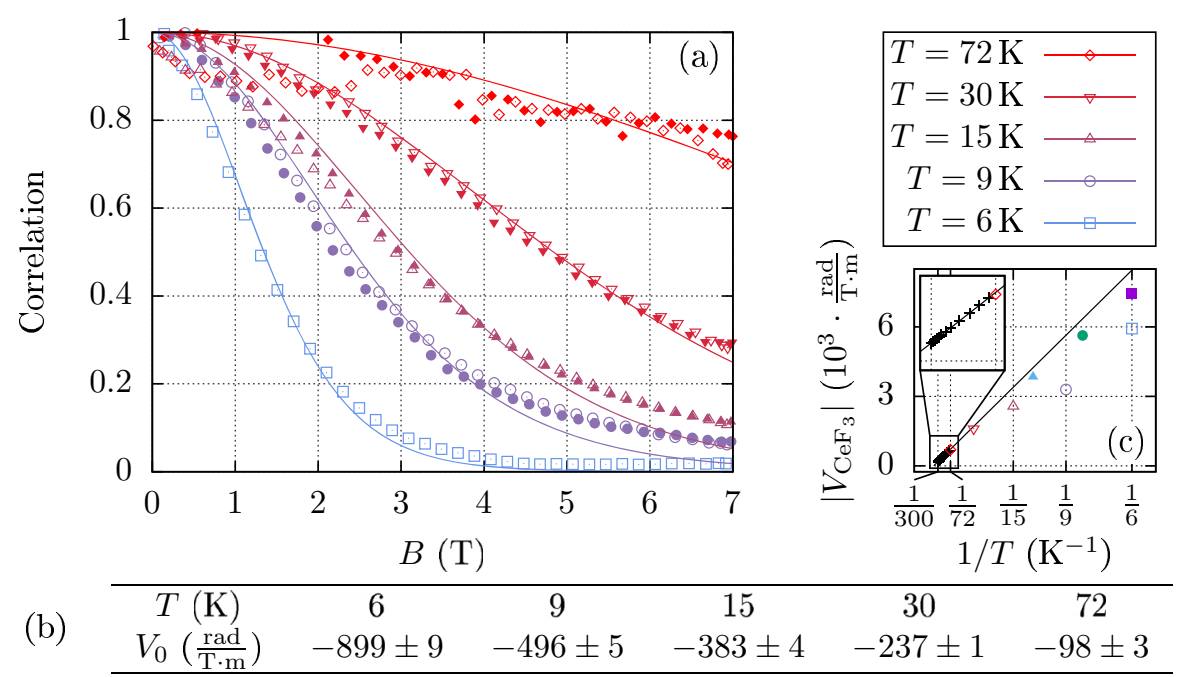

Fig. 5. (a) Correlations of the transmission speckle for a sample consisting of $3.8 \mathrm{vol} \% \mathrm{TiO}_{2}$ and $5.3 \mathrm{vol} \% \mathrm{CeF}_{3}$ solved in $90.9 \mathrm{vol} \%$ glycerol as a function of the magnetic field for different temperatures. Open symbols: increasing field, correlation with the first image; filled symbols: decreasing field, correlation with the last image. (For $T=6 \mathrm{~K}$, only the measurement with increasing field was evaluated since problems with the cryostat occurred during the decrease of the field.) The decorrelations are fitted (solid lines) as in Figure 4. (b) Measured Verdet constant $V_{0}$ for the different temperatures. (c) Recalculated bulk Verdet constant of $\mathrm{CeF}_{3}$ $V_{\mathrm{CeF}_{3}}$ (using the measured $E_{V}$ at $72 \mathrm{~K}$ for all temperatures) as a function of the inverse temperature (open symbols). For comparison the high temperature dependency of the Verdet constant (between $77 \mathrm{~K}$ and $300 \mathrm{~K}$ ) measured in reference [16] (black crosses) is plotted and fitted by $V \propto 1 / T$ (solid black line) and the low temperature data measured in Figure 6 are shown (filled symbols).

Verdet constant of $\mathrm{CeF}_{3} V_{\mathrm{CeF}_{3}}$ by knowing the volume fractions and density of the different materials. $V_{\mathrm{CeF}_{3}}$ is plotted as a function of inverse temperature in Figure 5c. The values are normalized by the value of $E_{V}$ for the high temperature measurement $(T=72 \mathrm{~K})$. For comparison we plotted the high temperature dependency of the Verdet constant (between $77 \mathrm{~K}$ and $300 \mathrm{~K}$ ) measured by Leycuras et al. [16] (black crosses, zoom in).

To obtain low temperature bulk values of the Faraday rotation of $\mathrm{CeF}_{3}$ a bulk c-oriented single crystal of $\mathrm{CeF}_{3}$ ( $L=1 \mathrm{~mm}$ from E-Crystal Co., Ltd., Osaka, Japan) was measured. A $532 \mathrm{~nm} \mathrm{CW}$-laser was passed through a polarizer and an analyzer with the bulk crystal plate in between inside the cryostat and the transmitted light was recorded with a photodiode. The magnetic field was raised from $0 \mathrm{~T}$ to $18 \mathrm{~T}$ for temperatures of $6 \mathrm{~K}, 8 \mathrm{~K}$ and $12 \mathrm{~K}$. The rotation angle $\beta$ is plotted in Figure 6 versus the applied field. We fitted the data with equation (3) to obtain the bulk Verdet constant $V_{0}$ for each temperature. The Verdet constant for the three temperatures are plotted in Figure 5c for comparison. They fall almost on the Leycuras et al. [16] data fit $\left(V_{0} \propto \frac{1}{T}\right)$ extrapolated for low temperatures.

\subsection{Field dependency of the Faraday rotation of $\mathrm{CeF}_{3}$}

In a second step we characterize the Faraday rotation in multiple scattering samples made of $\mathrm{CeF}_{3}$ and glycerol mixtures up to $B=18 \mathrm{~T}$ at low temperatures $(T=8 \mathrm{~K}$ 


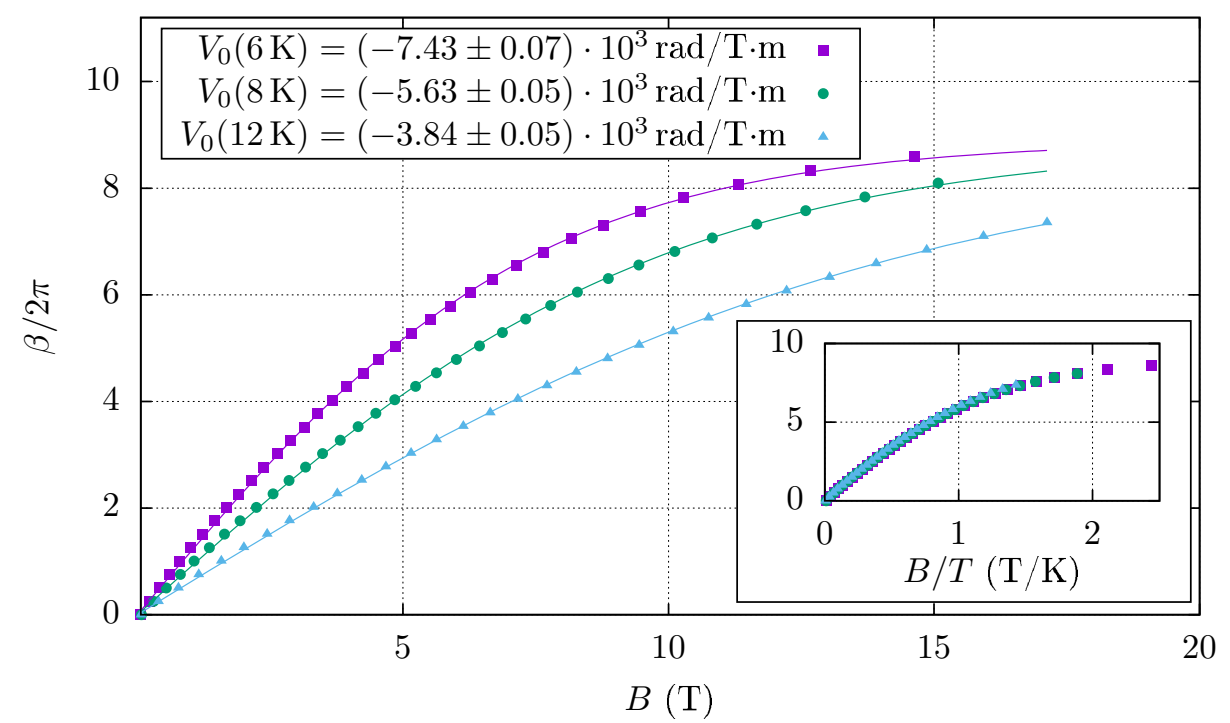

Fig. 6. Faraday rotation angle $\beta$ of a bulk crystal slab shaped sample $(L=1 \mathrm{~mm})$ as a function of magnetic field at 6,8 and $12 \mathrm{~K}$. A $532 \mathrm{~nm}$ CW-laser was used for illumination. The data were fitted with equation (3) to obtain the $B=0$ bulk Verdet constant $V_{0}$ and the saturation parameter $\alpha$ (solid lines). For all three temperatures, $\alpha=(0.81 \pm 0.02) \mathrm{K} / \mathrm{T}$ and $\kappa=(5.57 \pm 0.02) \cdot 10^{4} \mathrm{rad} \cdot \mathrm{m}^{-1}$. The inset shows that all the data collapse on a single curve when plotted as a function of $B / T$. The Verdet constant is proportional to the derivative of the curve and shows a saturation for high $B / T$ ratios.

and $9 \mathrm{~K}$ ). The measurements are carried out in the same magnet as the CBC measurements which has a vertical bore. This increases the instabilities of the measurement. Furthermore the higher fields require longer times to reach the full magnetic field $(18 \mathrm{~T})$ and thus a better stability is necessary. Moreover larger fields lead to larger forces on the setup and thus to movements. To overcome these stability issues a new measurement protocol is introduced.

The correlations are measured in $\Delta B=1 \mathrm{~T}$ field steps to shorten the measurement time. This also reduces the decorrelation due to movements of the setup in the field. Furthermore, the Faraday rotation of the windows becomes negligible. For one specific sample and if no saturation of the Faraday rotation is present, the speckle decorrelation should only depend on $\Delta B=B-B_{0}$ and not on $B_{0}$. A saturation leads to a weaker correlation decay for a given $\Delta B$ at higher fields $B_{0}$, and thus to lower $V$ as a result of the fit of equation (4).

We measured $V$ via the correlation decay for three samples with different filling fraction of $\mathrm{CeF}_{3}\left(f_{\mathrm{CeF}_{3}}=16.8 \mathrm{vol} \%\right.$ at $T=9 \mathrm{~K}, f_{\mathrm{CeF}_{3}}=20.7 \mathrm{vol} \%$ at $T=8 \mathrm{~K}$ and $f_{\mathrm{CeF}_{3}}=20.2 \mathrm{vol} \%$ at $T=9 \mathrm{~K}$ ). To compare the different measurements we normalize the absolute value of $V$ with $f_{\mathrm{CeF}_{3}}$ and multiply by the temperature $T$. This value now equals $\kappa \alpha$ from equation (2). The data are plotted in Figure 7 against the starting field $B_{0}$ of the field steps normalized by the temperature $T$. Note that the data overlay (within errors) although they were measured for somewhat different filling fractions and at different temperatures. The normalized Verdet coefficient clearly decreases for higher fields. First of all this indicates a saturation process. But even for high fields a decay of the correlation is still visible. Thus higher fields still lead to an enhanced Faraday rotation and it is worth to raise the field to $B=18 \mathrm{~T}$ to destroy reciprocity on even smaller paths. The data are fitted with equation (2) (solid black line) to obtain the material dependent constant 


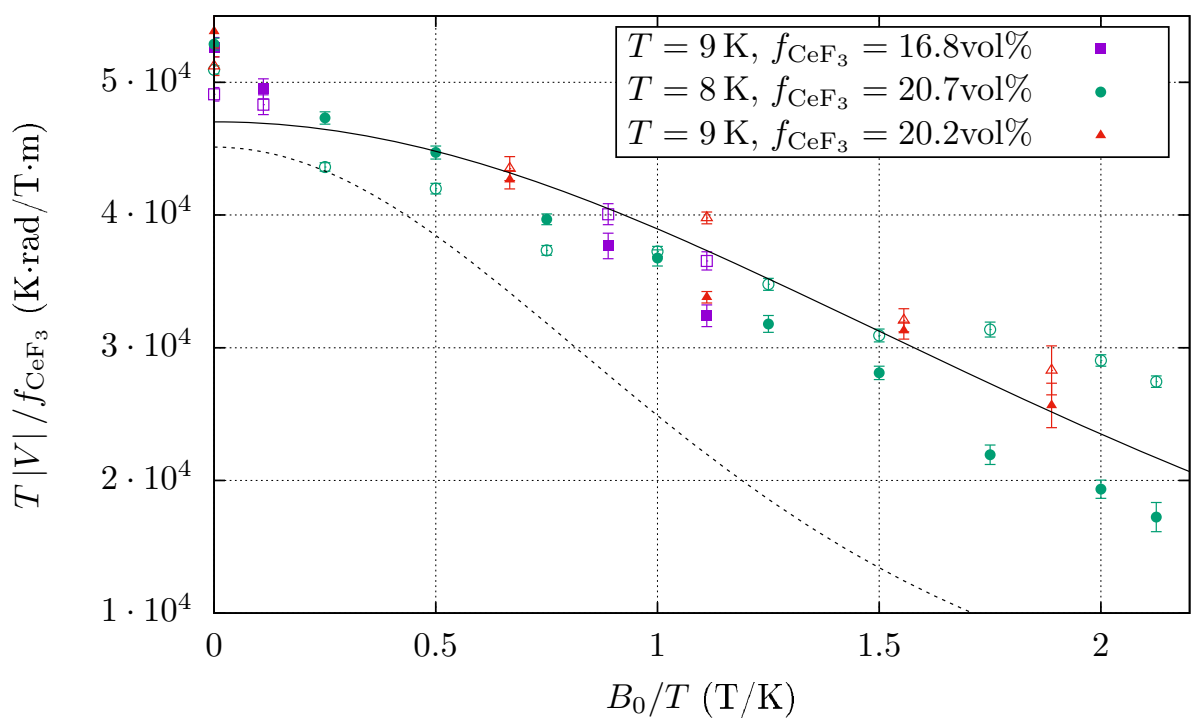

Fig. 7. Measurement of the saturation of the Faraday rotation of $\mathrm{CeF}_{3}$ for three different sample mixtures of $\mathrm{CeF}_{3}$ and glycerol: from correlation measurements such as in Figure 4 the Verdet constant $V$ is obtained for different $\Delta B=1 \mathrm{~T}$ field steps starting at $B_{0}$. Increasing field (open symbols) and decreasing field (closed symbols). $V$ is multiplied by $T / f_{\mathrm{CeF}_{3}}$ to fit all data together with equation 2 (solid black line). From the fit we obtained $\kappa \alpha=(4.70 \pm 0.09) \cdot 10^{4} \mathrm{~K} \cdot \mathrm{rad} \cdot \mathrm{T}^{-1} \mathrm{~m}^{-1}$ and $\alpha=(0.44 \pm 0.02) \mathrm{K} / \mathrm{T}$. For comparison equation 2 is plotted with the bulk $\mathrm{CeF}_{3}$ values for $\kappa$ and $\alpha$ (dashed black line).

$\kappa \alpha=(4.70 \pm 0.09) \cdot 10^{4} \mathrm{~K} \cdot \mathrm{rad} \cdot \mathrm{T}^{-1} \mathrm{~m}^{-1}$. We further plotted equation (2) using the bulk $\mathrm{CeF}_{3}$ values for $\kappa$ and $\alpha$ measured in Figure 6 (black dashed line). Again the experimental value is larger than the bulk value, consistent with Erbacher et al. [4]. Note that the material constant $\alpha=(0.44 \pm 0.02) \mathrm{K} / \mathrm{T}$ responsible for the field saturation is smaller than the bulk value. This discrepancy needs further investigations. Nevertheless, the bulk and multiple scattering data are still in qualitative agreement.

Figures 3, 6 and 7 show the first quantification of the Faraday rotation of $\mathrm{CeF}_{3}$ up to $18 \mathrm{~T}$ at low temperature. We observe a decrease of the Verdet constant for large magnetic field to temperature ratios, and thus a saturation of the Faraday rotation of $\mathrm{CeF}_{3}$.

\section{Conclusion}

In this paper we characterized the Faraday rotation of $\mathrm{CeF}_{3}$ down to $T=6 \mathrm{~K}$ and up to $B=18 \mathrm{~T}$. Moreover we showed that $\mathrm{CeF}_{3}$ can be used as a potential material to increase the Faraday effect in strongly scattering samples. By mixing it with higher refractive index materials (e.g. $\mathrm{TiO}_{2}$ ) it might be a candidate to manipulate reciprocity in localizing samples, if they are found.

Although the $\mathrm{CBC}$ is a direct measure of the coherent part of the light in multiple scattering samples and reciprocity can be easily accessed, destruction of the cone can not be realized in strongly scattering samples $k l^{*}<10$ with $\mathrm{CeF}_{3}$ using the available setup. Close to the mobility edge of Anderson localization, the samples have an even higher scattering strength. This means that in order to quantify the strength of Faraday rotation and to reach the breakdown of reciprocity in such samples 
in backscattering geometry, materials with a higher Verdet constant or a setup in stronger fields would be necessary.

Moreover, we present an alternative method, the correlation measurement of the transmission speckle, which allows a characterization of the Faraday rotation also in stronger scattering powders. The question whether a breakdown of reciprocity (as an interesting application for manipulation of Anderson localization) can be associated with the destruction of the speckle correlation in transmission remains open. One could argue that only photons traveling on reciprocal paths which fit inside the sample of size $L$ may localize (if the localization length is smaller than or comparable to $L$ ). Transmission speckle patterns are governed by similar path lenghts (of order $\left.L^{2} / \ell^{*}\right)$. Thus a breakdown of reciprocity in the helicity conserving channel might be expected to occur when the speckle correlation in transmission geometry has decayed. If potentially localizing samples are found we suggest time of flight experiments in high magnetic fields to distinguish between Anderson localization and other effects such as absorption and fluorescence.

We acknowledge support by the Deutsche Forschungsgemeinschaft (DFG), the Center for Applied Photonics (CAP), University of Konstanz and the Schweizerischer Nationalfonds (SNF).

\section{References}

1. M.P. Van Albada, Ad Lagendijk, Observation of weak localization of light in a random medium, Phys. Rev. Lett. 55, 2692 (1985)

2. P.-E. Wolf, G. Maret, Weak localization and coherent backscattering of photons in disordered media, Phys. Rev. Lett. 55, 2696 (1985)

3. P.W. Anderson, Absence of diffusion in certain random lattices, Phys. Rev. 109, 1492 (1958)

4. F.A. Erbacher, R. Lenke, G. Maret, Multiple light scattering in magneto-optically active media, Europhys. Lett. 21, 551 (1993)

5. M. Fink, Time reversal in acoustics, Contemp. Phys. 37, 95 (1996)

6. G. Bayer, T. Niederdränk, Weak localization of acoustic waves in strongly scattering media, Phys. Rev. Lett. 70, 3884 (1993)

7. F. Jendrzejewski, K. Müller, J. Richard, A. Date, T. Plisson, P. Bouyer, A. Aspect, V. Josse, Coherent backscattering of ultracold atoms, Phys. Rev. Lett. 109, 195302 (2012)

8. G. Modugno, Anderson localization in Bose-Einstein condensates, Rep. Prog. Phys. 73, $102401(2010)$

9. E. Akkermans, G. Montambaux, Mesoscopic Physics of Electrons and Photons (Cambridge University Press, 2007)

10. D. Vollhardt, P. Wölfle, Self-consistent theory of Anderson localization, in Electronic Phase Transitions, edited by W. Hanke and Yu.V. Kopaev, Vol. 32 of Modern Problems in Condensed Matter Sciences (Elsevier, Amsterdam, 1992), Chap. 1, pp. 1-78

11. R. Lenke, G. Maret, Affecting weak light localization by strong magnetic fields, Phys. Scrip. 1993, 605 (1993)

12. T. Sperling, L. Schertel, M. Ackermann, G.J. Aubry, C.M. Aegerter, G. Maret, Can 3D light localization be reached in 'hite paint'? New J. Phys. 18, 013039 (2016)

13. S.E. Skipetrov, J.H. Page, Red light for anderson localization, New J. Phys. 18, 021001 (2016)

14. J.X. Zhu, D.J. Pine, D.A. Weitz, Internal reflection of diffusive light in random media, Phys. Rev. A 44, 3948 (1991)

15. S. Fiebig, C.M. Aegerter, W. Bührer, M. Störzer, E. Akkermans, G. Montambaux, G. Maret, Conservation of energy in coherent backscattering of light, Europhys. Lett. 81, 64004 (2008) 
16. C. Leycuras, H. Le Gall, M. Guillot, A. Marchand, Magnetic susceptibility and Verdet constant in rare earth trifluorides, J. Appl. Phys. 55, 2161 (1984)

17. Ad Buckingham, Pj Stephens, Magnetic optical activity, Ann. Rev. Phys. Chem. 17, 399 (1966)

18. M.J. Weber, Handbook of Optical Materials, Laser \& Optical Science \& Technology (CRC Press, Boca Raton, 2003)

19. M.J. Weber, Faraday rotator materials, Lawrence Livermore Natl. Lab. Rep. M-103 (1982)

20. David Lacoste, Diffusion de la lumière dans les milieux magneto-optiques et chiraux, PhD thesis (Université Joseph Fourier, Grenoble, 1999)

21. F.A. Erbacher, Licht-Vielfachstreuung in magneto-optisch aktiven Medien, $\mathrm{PhD}$ thesis (Universität Konstanz, Konstanz, 1992)

22. L. Schertel, Magneto-optical Faraday effect in multiple-scattering media, Master's thesis (Universität Konstanz, Konstanz, December 2014)

23. R. Lenke, R. Lehner, G. Maret, Magnetic-field effects on coherent backscattering of light in case of Mie spheres, Europhys. Lett. 52, 620 (2000)

Open Access This is an Open Access article distributed under the terms of the Creative Commons Attribution License (http://creativecommons.org/licenses/by/4.0), which permits unrestricted use, distribution, and reproduction in any medium, provided the original work is properly cited. 